\title{
Pour en lire plus : Environnement et santé : Passer de la conscience à l'acte
}

Étienne van Steenberghe

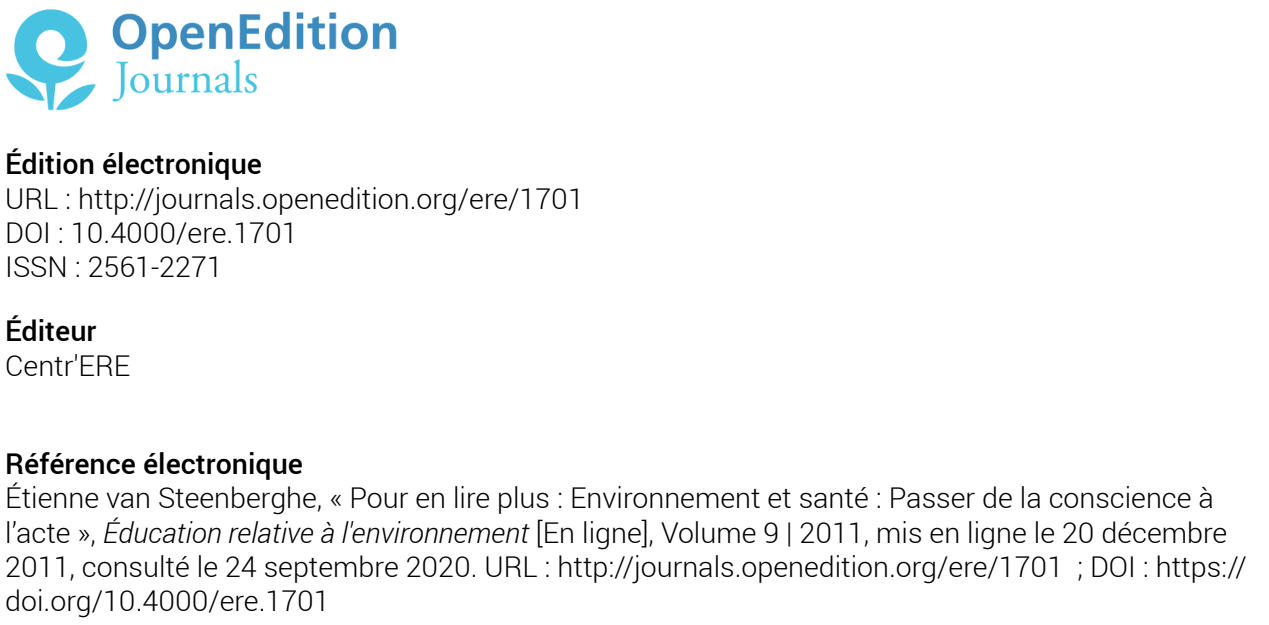

Ce document a été généré automatiquement le 24 septembre 2020. 


\title{
Pour en lire plus : Environnement et santé : Passer de la conscience à l'acte
}

\author{
Étienne van Steenberghe
}

\section{RÉFÉRENCE}

Groupement régional de promotion de la santé du Nord-Pas-De-Calais. (2009).

Environnement et santé : Passer de la conscience à l'acte. Contact Santé, Hors-série(3)

1 Malgré le titre de cette publication « Passer de la conscience à l'acte », on constate que la plupart des textes mettent peu en évidence la dimension éducative. On y trouve cependant des réflexions intéressantes sur le rapport aux liens entre santé et environnement et sur l'amélioration de l'agir à cet égard.

2 La relation que l'humain entretient avec son environnement évolue au fil du temps. La révolution industrielle et les nombreux progrès technologiques des dernières décennies ont contribué ainsi à développer notre emprise sur le monde, au point que nous en avons parfois oublié notre grande vulnérabilité. Ce numéro spécial de la revue Contact Santé rassemble les contributions de nombreux chercheurs et auteurs du milieu associatif autour de plusieurs questions d'actualité : Pourquoi est-il si complexe de comprendre les liens entre l'environnement et la santé ? Pourquoi l'environnement est-il devenu un fait politique majeur? Sommes-nous tous égaux face aux risques environnementaux? Pourquoi sommes-nous malades de notre environnement? Etc. Dans cette recension, nous nous limiterons à synthétiser les principaux articles qui abordent ces grandes questions et à mettre en lumière quelques réflexions sur l'agir relatif aux liens entre l'environnement et la santé.

3 En trame de fond, cette publication s'interroge sur la place que devrait occuper l'environnement dans le champ de la santé publique. Dans son éditorial intitulé «il nous faut changer de paradigme », Jean-Louis Salomez, professeur de santé publique de 
l'Université de Lille 2, reconnait toute la difficulté pour les différents acteurs sur le terrain, qu'ils soient issus de l'environnement ou de la santé, d'échanger entre eux. La question est alors de savoir comment articuler la santé publique dans le champ environnemental. Selon lui, le besoin de développer une approche pluridisciplinaire et collaborative est nécessaire si on considère que l'environnement est un des aspects majeurs dans un modèle global de santé.

Dans le premier article de la revue, Myriam Fritz Legendre, docteure en droit public, s'interroge sur toute la complexité d'établir des liens entre l'environnement et la santé. Si les relations entre les deux champs sont largement reconnues par les experts scientifiques, elles suscitent néanmoins des questions de plus en plus complexes et nombreuses. Les dégradations de l'environnement, qu'elles soient physiques et,ou sociales, peuvent constituer des agressions pour la santé. Pour y remédier, on met souvent en place des mesures ponctuelles sans pour autant prendre en compte toutes les interactions qui entrent en jeux. Lionel Charles, dans son article intitulé "Généalogies de l'environnement ", nous signale que cette complexité se double d'un aspect culturel qui tient au caractère particulier de notre relation au monde.

5 Michel Autès, chercheur au CNRS et vice-président responsable de la santé pour le Conseil régional Nord Pas-de-Calais en France, poursuit la réflexion des liens entre l'environnement et la santé sous un angle plus politique. Dans son texte, il reconnait que l'environnement est devenu un fait politique incontournable depuis que nous avons pris conscience de la fragilité de notre planète et des risques d'extinction de l'espèce humaine. Mais, il constate aussi que les vrais actes politiques tardent à suivre. On se contente trop souvent de belles déclarations. Les exemples sont particulièrement nombreux. Michel Autès avance trois éléments de réponse à cet «immobilisme ». Premièrement, la difficulté que nous avons à nous détacher du passé et du présent pour envisager un avenir différent. Deuxièmement, nos sociétés occidentales qui s'appuient sur les principes de concurrence et de compétition au lieu de favoriser l'émergence d'une collectivité basée sur la solidarité. Troisièmement, une conception souvent trop étroite de l'environnement qui ne prend pas suffisamment en compte nos rapports à autrui et à tout ce qui nous entoure. Dès lors, la prise de conscience d'un "nous » individuel et collectif semble être une sorte de préalable pour poser des actes.

La littérature montre que la salubrité de notre environnement comme facteur de bienêtre et de santé est inégalement répartie. Ces inégalités sont reconnues comme une forme d'injustice et suscitent l'action. Helga-Jane Scrawell et Isabelle Roussel de l'Université de Lille font référence à la notion américaine de la justice environnementale qui a pris naissance au cœur d'un mouvement social et populaire de lutte pour la prise en compte des inégalités socioenvironnementales. Dans certains pays comme la France, la justice environnementale s'est plutôt imposée à partir des études sur les inégalités sociales de santé et ne s'est dès lors pas inscrite a priori dans un mouvement de revendication. Pour ses deux auteurs, l'environnement doit être pensé en termes de justice et devenir synonyme d'une lutte sociale qui s'accompagne d'une réflexion sur les liens entre le bien-être et l'environnement. Une telle perspective nous semble des plus intéressantes si elle vise à accompagner les personnes dans leur capacité d'agir pour améliorer leur cadre de vie. Or, trop souvent, on reste dans des constats, sans passer aux actes.

7 Joël Spiroux, docteur en médecine générale et environnementale, nous parle de l'apparition d'un nouveau concept: les pathologies environnementales. Celui-ci fait 
suite à une augmentation du nombre de maladies et, ou d'affections pouvant être reliées à l'environnement. Le modèle d'une relation univoque de cause à effet n'est plus d'actualité, car il n'intègre pas la complexité des interactions environnementales (facteurs biologiques, physiques, chimiques, sociaux). Dès lors, Joël Spiroux estime qu'il nous faut changer notre regard et intégrer la nécessité de penser la complexité du monde qui nous entoure. Il s'inscrit ainsi dans la suite des auteurs précédents et partage le discours de Jean-Louis Salomez dans son éditorial.

Plusieurs autres textes de cette publication traitent d'enjeux environnementaux plus spécifiques tels que les préoccupations concernant la qualité de l'air et de l'eau, les craintes sur les organismes génétiquement modifiés et l'impact des changements climatiques sur notre santé. Chaque cas est illustré soit par des études collectives impliquant divers groupes de recherche, soit par des interventions exemplaires menées par le milieu associatif à l'échelle locale ou régionale. Certaines actions présentées dans cette revue insistent sur l'importance de s'appuyer sur les forces du milieu et la nécessité de susciter un dialogue qui favorise la diversité des solutions applicables. Cela semble d'autant plus important vu la complexité des liens entre l'environnement et la santé. Mais, il est intéressant de constater que la plupart des interventions environnementales qui font appel à la santé publique ont tendance - aujourd'hui encore - à s'appuyer sur des données épidémiologiques, alors que l'outil ne semble plus adapté face à la complexité des liens entre l'environnement et la santé. Un autre outil reste donc à découvrir.

9 La majorité des articles présentés dans ce numéro de la revue Contact Santé sont fort pertinents. Ils constituent un ensemble de petits textes illustrés par des pratiques associatives, des contenus qui donnent envie d'approfondir le sujet à travers d'autres lectures et,ou discussions.

\section{AUTEUR}

\section{ÉTIENNE VAN STEENBERGHE}

Coordonnateur du Réseau francophone international de recherche en éducation relative à

l'environnement. Montréal, Canada 